\title{
DISTURBANCE DISTRIBUTION MATRIX COMPUTATION: NUMERICAL IMPROVEMENT.
}

\author{
Faisel UPPAL ${ }^{\S}$, Suzanne LESECQ*, Ron PATTON ${ }^{\S}$, Alain BARRAUD* \\ ${ }^{\S}$ Control \& Intelligent Systems Engineering, The University of Hull, Hull, HU6 7RX, UK. \\ f.uppal@hull.ac.uk,r.j.patton@hull.ac.uk, http://www.eng.hull.ac.uk/research/control \\ * Laboratoire d'Automatique de Grenoble, BP46, 38402 Saint Martin d'Hères Cedex - France \\ suzanne.lesecq,alain.barraud@inpg.fr, http://www.lag.inpg.fr
}

\begin{abstract}
:
Prompt detection and diagnosis of process malfunctions are strategically important due to economic and environmental demands required for industries to remain competitive in world markets. In this paper a new formulation of the computation of the disturbance and fault distribution matrices is suggested for Neuro-Fuzzy and De-coupling Fault Diagnosis Scheme (NFDFDS). NFDFDS is a multiple-model fault detection and isolation (FDI) approach of non-linear dynamic systems. In this approach, powerful approximation and reasoning capabilities of neuro-fuzzy models are combined with the de-coupling capabilities of optimal observers to perform reliable fault detection and isolation. For determination of distribution matrices in this case it is shown that a least-squares approach is the most efficient compared with any other non-linear optimization technique. Copyright (C) 2005 IFAC
\end{abstract}

Keywords: Fault Detection and Isolation (FDI), Neuro-fuzzy (NF), multiple-model observer, least-squares (LS) minimisation, optimization.

\section{INTRODUCTION}

To maintain a high level of performance, safety and reliability in control systems the errors, component faults and abnormal system operation must be detected promptly. The source and severity of each malfunction must be diagnosed so that corrective action can be taken quickly. Most real application systems suffer from disturbances and noise and varying operating conditions, leading to a challenging modelling requirement. The modelreality differences have an associated 'uncertainty' and this usually means that the robust linear and nonlinear estimators need to be considered.

A lot of model-based FDI methods rely on a linear state-space model of the system. However, for nonlinear systems, the standard approach is to linearise the process model around an operating point and make use of model-based methods derived from linear systems theory. However, linearisation does not provide a good model for the processes with strongly non-linear behaviour.

It is well known that solutions to the robustness problem of FDI for non-linear dynamic systems depend upon reliable discrimination between the effect of uncertain model behaviour and faults (Chen \& Patton, 1996; 1999).

The robust observer approaches, particularly the 'unknown input observer' (UIO) are undoubtedly the most commonly used FDI methods in the present context. These linear systems methods can, to some extent compensate for model uncertainty thus increasing the reliability of fault detection and isolation. The model-reality mismatch is represented by the so-called 'unknown input'. Most of the work on the UIO has been directed towards linear systems. However, there are some observer-based approaches 
for certain classes of non-linear systems but the systems that can be represented by these non-linear observers are limited to a few standard types of nonlinearity (Chen \& Patton, 1996; 1999). Furthermore, the non-linear observer approach can only be used when the non-linear dynamics are known with sufficient confidence; this is rarely the case for real system applications.

In this context, the Neuro-Fuzzy (NF) methods are known to overcome some of the problems faced by the model-based techniques. NF models combine the approximation capability of Neural Networks with the reasoning of fuzzy logic (Brown \& Harris, 1995; Jang, Sun \& Mizutani, 1997). Although a trade-off exists between modelling accuracy and reasoning capabilities of NF schemes, the required structure is application dependent (Brown \& Harris, 1995; Jang, Sun \& Mizutani, 1997). This combination gives rise to a powerful form of multiple-model approximation that is especially attractive when non-linear systems are considered and for which only global modelling can be valid. Here, our interest is in modelling nonlinear systems for the purpose of detecting and isolating system faults and hence a degree of transparency is required, to localise faults, understand symptoms etc.

This NF model is then associated with local optimal observers based on the unknown input observer principle. This leads to the so-called Neuro-Fuzzy and De-coupling Fault Diagnosis Scheme (NFDFDS) which will be summarized in section 2 . Section 3 gives a résumé of the main steps involved in the NFDFDS. Note that one of the most important steps of this scheme is the determination of the fault and disturbance distribution matrices. As it is recalled in Section 4, this computation can be achieved with different techniques. In this paper (Section 5), a new approach based on the reformulation of the problem as a least-squares problem is given. It facilitates an improvement in the optimality (degree of disturbance de-coupling) of the disturbance matrix computation and thereby reducing the computational cost. Sections 6 and 7 are devoted to the comparision of the results achieved with the LS formulation with previously used optimisation techniques. The application study considered is the DAMADICS Benchmark Problem described in Section 6 (DAMADICS Benchmark definition, ver.1.0. 2003).

\section{NEURO-FUZZY AND DE-COUPLING FAULT DIAGNOSIS SCHEME (NFDFDS)}

In real applications the disturbances, noise and modelling errors must all be taken into account. The FDI scheme outlined here (Neuro-Fuzzy and Decoupling Fault Diagnosis Scheme, NFDFDS) employs multiple local optimal observers designed according to minimum state estimation variance (Uppal \& Patton, 2004). Through this approach, the approximation and reasoning capabilities of neurofuzzy models are combined with the de-coupling capabilities of optimal observers to perform reliable fault detection and isolation. The structure of the FDI scheme is transparent as compared to the NN 'black box' method and it can also deal with non-linear systems in contrast to model-based methods.

The proposed FDI scheme (Fig. 1) consists of two main parts: a bank of $(M+1) \times N$ NF-generated decoupling observers (based on the unknown input observer principle) and a diagnostic logic unit. The first part generates a set of residuals $\left(r_{k}^{0}, \ldots, r_{k}^{M}\right)$ at the $k^{\text {th }}$ sampling instant, in the form of a structured residual set (Chen \& Patton, 1999), whilst the second part performs an analysis of the residuals to determine the nature and location of the faults. In this scheme, each residual is designed to be sensitive to a subset of faults or the residuals are designed to have specific behaviour (sensitivity) in response to the different faults. Ideally, each residual is sensitive to all but one fault. The set of all such residuals is known as a 'Generalized Residual Set' based on the generalized observer scheme of Frank, (Chen \& Patton, 1999; Patton, Frank \& Clark, 1989).

Each of the $M+1$ 'Fault Diagnosis Observers' in Fig. 1 is a non-linear system comprising $N$ linear (integrated by fuzzy fusion) sub-observers each one corresponding to a different operating point of the process. The inputs to each observer are the process inputs and outputs, $u_{k}$ and $y_{k}$, respectively. The number of sub-observers depends on the number of operating points needed to achieve required approximation. Their outputs are combined by fuzzy fusion to generate the output estimates. The set of residuals comprises the set of differences between the actual and estimated outputs. The set of fuzzy observers together with the NF multiple-model and diagnostic logic form the new scheme called the 'NFDFDS'.

The computational problem increases further with the increase in the number of fuzzy rules resulting in a larger bank of observers. However, these subobservers are linear which makes it possible to use the well-developed linear theory. Moreover, the observer design is swift and routine, once the model identification is achieved and the fault distribution matrices are determined.

\section{NFDFDS FORMULATION}

In NFDFDS the sub-observers are based on the local linear models with fault and disturbance as described below:

$$
\begin{aligned}
& x_{k+1}^{l}=A_{k}^{l} x_{k}^{l}+B_{k}^{l} u_{k}^{c}+E_{k}^{l} d_{k}+F_{1 k}^{l} f_{k}+w_{1 k}^{l} \\
& y_{m k}^{l}=C_{k}^{l} x_{k}^{l}+w_{2 k}^{l}
\end{aligned}
$$

where $u_{k}^{c} \in \mathbf{R}^{r}, \quad x_{k}^{l} \in \mathbf{R}^{n}, \quad y_{m k}^{l} \in \mathbf{R}^{m}$ are the input, state and output vectors for the $l$-th consequent linear model respectively. Each entry of $f_{k} \in \mathbf{R}^{g}$ corresponds to a specific fault while $d_{k} \in \mathbf{R}^{q}$ stands for the disturbance vector. $A_{k}^{l}, B_{k}^{l}$ and $C_{k}^{l}$ are the system matrices with appropriate dimensions. The fault distribution matrix $F_{1 k}^{l} \in \mathbf{R}^{n \times g}$ represents the effect of input and component faults on the system. $w_{1 k}^{l}$ and $w_{2 k}^{l}$ are supposed to be independent zero- 
mean white noise sequences with correlation matrices $Q_{k}$ and $R_{k}$, assumed to be known.

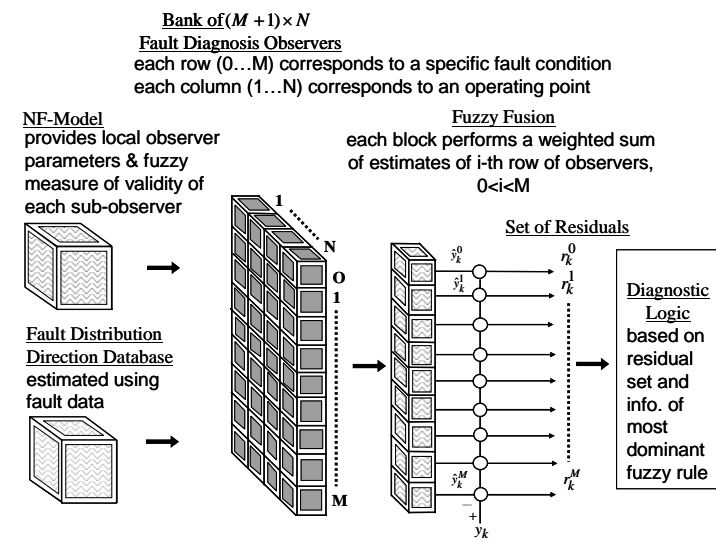

Fig. 1: Neuro-fuzzy decoupling fault diagnosis scheme

The global model can be described by fuzzy IFTHEN rules that represent the local linear models of the non-linear system in the following form:

Rule- $I,(I=1,2, \ldots, N)$ :

$$
\text { IF } u_{k}^{a} \text { is } \mathrm{M}_{l} \text { THEN } y_{m_{k}}=y_{m_{k}}{ }^{l}
$$

The global output of the fuzzy model is obtained by de-fuzzification (weighted sum of the outputs of the sub-models) as:

$$
y_{m_{k}}=\sum_{l=1}^{N} \alpha^{l}\left(u_{k}^{a}\right) y_{m k}^{l}
$$

In the above equation $\alpha^{l}\left(u_{k}^{a}\right) \forall l=1, \ldots, N$ is the firing strength of $l$-th rule, which depends on the antecedent variable $u_{k}^{a} . \mathrm{M}_{l}$ is a fuzzy set and $N$ is the total number of linear sub-models.

For residual generation, for each sub-model an optimal disturbance de-coupling sub-observer is designed as described in detail in (Uppal \& Patton 2004; Uppal, Patton \& Witczak, 2004). Note that the determination of the fault and disturbance distribution matrices constitutes one of the foremost steps in the NFDFDS scheme. The classical approaches used in order to compute these matrices are summerized hereafter.

\section{FAULT AND DISTURBANCE DISTRUBUTION MATRICES: CLASSICAL APPROACHES}

The term $E_{k}^{l} d_{k}$ in the $l$-th sub-system description (Eq. (1)) represents model uncertainty and other factors affecting reliability of the fault detection. This term may be used to represent a number of different types of disturbances acting upon the system. Moreover, the distribution matrix $E_{k}^{l}$ may be time-varying.

In order to determine the distribution matrices a number of techniques can be employed including 'Evolutionary Algorithms' (Dasgupta \& Michalewicz, 1997), 'Gradient Based Methods' (Hagan, Demuth \& Beale, 1996) or random search. The Augmented Observer described in (Chen \& Patton, 1999) is often not suitable as the necessary existence condition of this observer is that $\operatorname{rank}(C)=n$, where $n$ is the number of system states. This limits the use of this technique, as it requires that the system has $n$ independent measurements.

The underlying problem is to determine the disturbance distribution matrix $E_{k}^{l} \in \mathbf{R}^{n \times q}$ and the fault distribution matrix $F_{1 k}^{l}$ for $l=1, \ldots N$.

Actually, this problem boils down to the determination of the disturbance term $d_{1 k}$ in Eq. (4) for the normal and faulty system operations:

$$
\begin{aligned}
x_{k+1}^{l} & =A_{k}^{l} x_{k}^{l}+B_{k}^{l} u_{k}^{c}+d_{1 k}^{l} \\
y_{m k}^{l} & =C_{k}^{l} x_{k}^{l}+w_{2 k}^{l}
\end{aligned}
$$

where $d_{1 k}^{l}=E_{k}^{l} d_{k}+F_{1 k}^{l} f_{k}+w_{1 k}^{l}=E_{1 k}^{l} f_{1 k}^{l}$

This corresponds to the possible solutions for $E_{k}^{l}$ and $F_{1 k}^{l} f_{k}$ respectively:

(Witczak, et al., 2003) describes an approach for unknown input estimation for the following class of systems:

$$
\begin{aligned}
& x_{k+1}=g\left(x_{k}\right)+h\left(u_{k}\right)+d_{1 k} \\
& y_{k+1}=C_{k+1} x_{k+1}
\end{aligned}
$$

where $g(\cdot)$ and $h(\cdot)$ are continuous and differentiable non-linear functions of $x_{k}$ and $u_{k}$ at the $k^{\text {th }}$ sampling instant, respectively.

Notice that this approach assumes a constant disturbance distribution direction for the system described by Eq. (5).

In NFDFDS structure a method is described for determining an individual distribution direction for each sub-model (Uppal \& Patton 2004; Uppal, Patton \& Witczak 2004). This method considers the matrices $E_{1 k}^{l}$ directly i.e. the fault distribution matrix $E_{1 k}^{l}$ is considered to be constant over time for each fault scenario. Therefore, for a particular fault scenario $i$, an unconstrained non-linear optimisation task can be performed with the objective to minimise an unconstrained multivariable function:

$$
\tilde{E}_{1}^{i}=\arg \min _{E_{1}^{i}} f\left(E_{1}^{i}\right)
$$

where $f\left(E_{1}^{i}\right)=\frac{1}{n d-1} \sum_{k=0}^{n d-1} \varepsilon_{k}^{T} \varepsilon_{k}, \varepsilon_{k}=y_{k}-\hat{y}_{k}$ and $\hat{y}_{k}$ is a function of $E_{1}^{i}$.

The Nelder-Mead Simplex Method (Walsh, 1975) has been first used for convenience to compute the distribution matrices $E_{1 k}^{l}$ directly. In the sequel, this problem will be rewritten as a Least-Squares (LS) problem, leading to a much more efficient computational tool.

\section{LEAST-SQUARES SOLUTION}

In the following three cases are presented as an improvement to determine the disturbance terms, 
which enable vectors $d_{1 k}^{l}$ to be computed. They are all based on LS formulation.

CASE I. Consider that the individual disturbances sources $d_{1 k}^{l}, l=1, \ldots, N$ correspond to each submodel. The problem of unknown input estimation can be viewed as an unconstrained optimisation task of the form:

$$
\begin{aligned}
& \hat{\bar{d}}_{1 k}=\arg \min _{\bar{d}_{1 k}}\left\|\varepsilon_{k+1}\right\|_{2}^{2} \\
& \text { where } \bar{d}_{1 k}=\left[\left[d_{1 k}^{1}\right]^{T}\left[d_{1 k}^{2}\right]^{T} \ldots\left[d_{1 k}^{N}\right]^{T}\right]^{T} \in \mathbf{R}^{n N}
\end{aligned}
$$

where $\varepsilon_{k+1}=y_{k+1}-\hat{y}_{k+1}$ is the output error, $\hat{y}_{k+1}=\sum_{l=1}^{N} \alpha^{l} y_{m k+1}^{l}$ is the process output estimation, $\alpha^{l}$ is the weight held on to each model $l$ and $y_{m_{k+1}}^{l}$ has been defined in Eq. (1). A few manipulations lead to:

$$
\begin{aligned}
\varepsilon_{k+1} & =y_{k+1}-\sum_{l=1}^{N} \alpha^{l} y_{m k+1}^{l} \\
& =y_{k+1}-\sum_{l=1}^{N} \alpha^{l}\left(C_{k+1}^{l} x_{k+1}^{l}+w_{2 k+1}^{l}\right) \\
& =y_{k+1}-\sum_{l=1}^{N} \alpha^{l}\left(C_{k+1}^{l}\left(A_{k}^{l} x_{k}^{l}+B_{k}^{l} u_{k}^{c}+d_{1 k}^{l}\right)+w_{2 k+1}^{l}\right) \\
& =\beta_{k+1}-\sum_{l=1}^{N} \alpha^{l} C_{k+1}^{l} d_{1 k}^{l}
\end{aligned}
$$

where

$$
\beta_{k+1}=\left(y_{k+1}-\sum_{l=1}^{N} \alpha^{l}\left(C_{k+1}^{l}\left(A_{k}^{l} x_{k}^{l}+B_{k}^{l} u_{k}^{c}\right)+w_{2 k+1}^{l}\right)\right) \in \mathbf{R}^{m \times n N} .
$$

The optimisation problem (7) is thus solved using a Least-Square (LS) minimisation:

$$
\hat{\bar{d}}_{1 k}=\arg \min _{\bar{d}_{1 k}}\left[f\left(\overline{d_{1 k}}\right)=\left\|K_{k+1} \bar{d}_{1 k}-\beta_{k+1}\right\|_{2}^{2}\right]
$$

with

$K_{k+1}=\left[\begin{array}{llll}\alpha^{1} C_{k+1}^{1} & \alpha^{2} C_{k+1}^{2} & \cdots & \alpha^{N} C_{k+1}^{N}\end{array}\right] \in \mathbf{R}^{m \times n N}, m \leq n N$.

If $\operatorname{rank}\left(K_{k+1}\right)=m$, the solution is traditionally expressed through the normal equations:

$$
\overline{d_{1 k}}=\left(K_{k+1}^{T} K_{k+1}\right)^{-1} K_{k+1}^{T} \beta_{k+1}
$$

Eq. (9) can be solved efficiently using an orthogonal factorisation of matrix $K_{k+1}^{T}$ (Lawson \& Hanson, 1995), leading to a solution $\hat{\bar{d}}_{1 k}$ with minimum L-2 norm:

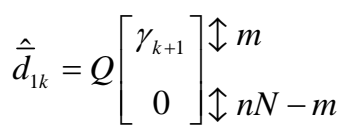

where:

$$
\begin{aligned}
& U^{T} \gamma_{k+1}=\beta_{k+1} \\
& Q K_{k+1}^{T}=\left[\begin{array}{c}
U \\
\ldots \\
0
\end{array}\right] \text { with } U \in \mathbf{R}^{m \times m} \text { being an upper }
\end{aligned}
$$

triangular matrix.

Note that the condition $\operatorname{rank}\left(K_{k+1}\right)=m$ can be relaxed. More details about this technique can be found in (Lawson \& Hanson, 1995).
From the estimates $\hat{\bar{d}}_{1 \mathrm{k}}$ at the different instants $k=1, \ldots, n d$, the determination of matrix $E_{1 k}^{i}$ is straight forward as detailed in (Chen \& Patton, 1999).

CASE II. It is now assumed that the overall disturbance in the system (1) can be de-coupled by only the $\mathrm{i}^{\text {th }}$ sub-model corresponding to the most dominant rule, i.e. $d_{1 k}^{l}=0 \forall l \neq i, l=1, \ldots, N$. The problem of unknown input estimation is again an optimisation task such that:

$$
\hat{\bar{d}}_{1 k}=\arg \min _{d_{1 k}^{i}}\left\|\varepsilon_{k+1}\right\|_{2}^{2}, d_{1 k}^{i} \in \mathbf{R}^{n}
$$

where:

$$
\begin{aligned}
\varepsilon_{k+1} & =y_{k+1}-\sum_{l=1}^{N} \alpha^{l} y_{m k+1}^{l} \\
& =y_{k+1}-\sum_{\substack{l=1 \\
l \neq i}}^{N} \alpha^{l} y_{m k+1}^{l}-\alpha^{i} y_{m k+1}^{i} \\
& =\beta_{k+1}-\alpha^{i} C_{k+1}^{i} d_{1 k}^{i} \\
\beta_{k+1} & =\left(y_{k+1}-\sum_{\substack{l=1 \\
l \neq i}}^{N} \alpha^{l} y_{m k+1}^{l}-\alpha^{i}\left(C_{k+1}^{i}\left(A_{k}^{i} x_{k}^{i}+B_{k}^{i} u_{k}^{c}\right)+w_{2 k+1}^{i}\right)\right) \in \mathbf{R}^{m}
\end{aligned}
$$

As described above, the solution with the minimum L-2 norm is computed via LS methods (Lawson \& Hanson, 1995):

$$
\hat{\bar{d}}_{1 k}=\arg \min _{d_{1 k}^{i}}\left[f\left(d_{1 k}^{i}\right)=\left\|K_{k+1} d_{1 k}^{i}-\beta_{k+1}\right\|_{2}^{2}\right]
$$

with $K_{k+1}=\left[\alpha^{i} C_{k+1}^{i}\right] \in \mathbf{R}^{m \times n}, m \leq n$.

CASE III. The fault distribution matrix $E_{1 k}^{l}$ is supposed to be constant over time for each fault scenario. Therefore, for a particular fault scenario $i$, (1) becomes:

$$
\left\{\begin{array}{l}
x_{k+1}^{l}=A_{k}^{l} x_{k}^{l}+B_{k}^{l} u_{k}^{c}+E_{1}^{l}(i) f_{1 k}^{i} \\
y_{m k}^{l}=C_{k}^{l} x_{k}^{l}+w_{2 k}^{l}
\end{array}\right.
$$

where $i=1, \ldots, M$ is the fault scenario number (see Fig. 1). For fault scenario $i$, the output error $\varepsilon_{k+1}^{i} \in \mathbf{R}^{m}$ is:

$$
\begin{aligned}
\mathcal{E}_{k+1}^{i}= & y_{k+1}-\sum_{l=1}^{N} \alpha^{l} y_{m k+1}^{l} \\
= & y_{k+1}-\sum_{l=1}^{N} \alpha^{l}\left(C_{k+1}^{l} x_{k+1}^{l}+w_{2 k+1}^{l}\right) \\
= & \left(y_{k+1}-\sum_{l=1}^{N} \alpha^{l}\left(C_{k+1}^{l}\left(A_{k}^{l} x_{k}^{l}+B_{k}^{l} u_{k}^{c}\right)+w_{2 k+1}^{l}\right)\right) \\
& \quad-\left(\sum_{l=1}^{N} \alpha^{l} C_{k+1}^{l} E_{1}^{l}(i)\right) f_{1 k}^{i} \\
= & \beta_{k+1}-K_{k+1} \overline{E_{1}(i)} f_{1 k}^{i}
\end{aligned}
$$

with $\overline{E_{1}(i)}=\left[\begin{array}{llll}E_{1}^{1}(i)^{T} & E_{1}^{2}(i)^{T} & \ldots & E_{1}^{N}(i)^{T}\end{array}\right]^{T} \in \mathbf{R}^{n \times \times g}$

If one chooses $f_{1 k}^{i}$ as a scalar (which means $g=1$ ) (Chen \& Patton, 1999), then:

$$
\varepsilon_{k+1}^{i}=\beta_{k+1}-f_{1}^{i} \mathrm{~K}_{k+1} \overline{E_{1}(i)} .
$$

The optimisation problem that is solved now can be expressed as:

$$
\hat{E}_{1}(i)=\arg \min _{E_{1}(i)}\left[f\left(\overline{E_{1}(i)}\right)=\left\|\overline{\mathrm{K}} \overline{E_{1}(i)}-\bar{\beta}\right\|_{2}^{2}\right]
$$


where

$$
\begin{aligned}
& \overline{\varepsilon^{i}}=\left[\left(\varepsilon_{1}^{i}\right)^{T} \cdots\left(\varepsilon_{n d}^{i}\right)^{T}\right]^{T} \in \mathbf{R}^{m \cdot n d}, \\
& \overline{\mathrm{K}}=\left[\begin{array}{c}
f_{1}^{i} \mathrm{~K}_{1} \\
\vdots \\
f_{1 n d}^{i} \mathrm{~K}_{n d}
\end{array}\right] \in \mathbf{R}^{n d \cdot m \times n N}, \bar{\beta}=\left[\begin{array}{c}
\beta_{1} \\
\vdots \\
\beta_{n d}
\end{array}\right] \in \mathbf{R}^{m \cdot n d} .
\end{aligned}
$$

Note that it is quite evident that Eq. (16) can be solved using a LS method, as previously shown.

\section{DESCRIPTION OF THE APPLICATION}

These results are now applied to a benchmark described in (DAMADICS Benchmark definition, ver.1.0. 2003). This study is based on a non-linear electro-pneumatic flow control valve at the Lublin sugar plant in Poland. This factory produces 50,000 tonnes of sugar annually (DAMADICS RTN Information Web site, 2003). The plant consists of a large number of evaporization sub-processes and boiler houses, heaters and valves. Although it is not economically possible to monitor all the valves, the main idea is to apply FDI techniques to a few of the "critical" valves (as these also involve several important process variables) to devise a powerful means of monitoring the process itself. For the purpose of this paper one valve has been selected as shown in Fig. (2).

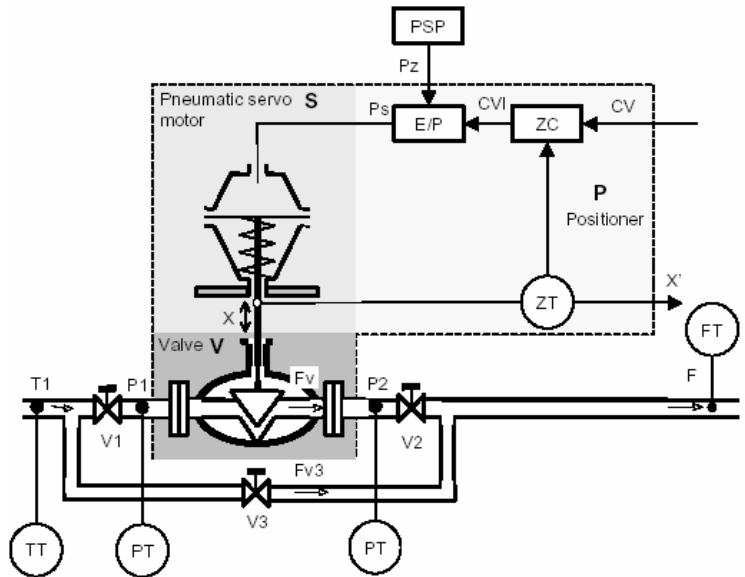

Fig. 2: Schematic diagram of the flow control valve

The process considered for FDI has four inputs and two outputs. The process inputs are the control value $\left(\mathrm{u}_{1}={ }^{\prime} c v^{\prime}\right)$, the inlet pressure $\left(\mathrm{u}_{2}={ }^{\prime} P 1 '\right)$, the outlet pressure $\left(\mathrm{u}_{3}={ }^{\prime} P 2\right.$ ') and the temperature $\left(\mathrm{u}_{4}={ }^{\prime} T\right.$ ' $)$, while the outputs are the stem displacement of the electro-pneumatic servomotor $\left(y_{1}=\right.$ ' $X_{s d}$ ') and the liquid flow through the valve $\left(y_{2}=' 1-F\right.$ '). A nonlinear SIMULINK model (DAMADICS Benchmark definition, ver.1.0. 2003) is used to generate the faulty data. A detailed fault description is given in the EC Framework 5 DAMADICS benchmark study definition referred above.

\section{RESULTS}

The fault scenario considered here corresponds to the valve clogging (referred as "Fault 1" in the DAMADICS benchmark). The fault distribution matrices $E_{1 k}^{l}$ are computed thanks to CASE III in section 5 . Their performances are compared with the matrices previously computed with a Nelder and
Mead method (Uppal \& Patton, 2004; Uppal, Patton \& Witczak, 2004).

Table 1 gives a comparison of the results achieved with both methods. The LS method reaches the unique solution (with minimum L-2 norm when necessary) while the Nelder and Mead technique does not produce a satisfactory result. Actually, the exit result is nothing but an intermediate result (even in the last case where the number of call to the function is equal to $10^{6}$ and the tolerance on the solution is $10^{-15}$ ) because the optimization task exits with the maximum number of iteration being exceeded.

Moreover, in a computational cost point of view, the LS method is infinitly more efficient than the Nelder and Mead approach. This second advantage is of interrest when on-line re-computation of the fault distribution matrices are performed.

Lastly, the LS approach has shown that the optimization problem has potentially an infinity of solutions when the Least Squares matrix is numerically rank deficient. In this case, the Nelder and Mead optimization method cannot find in a proper way any minimum.

Note that in this example zero initial conditions were used. The choice of initial conditions does affect the result, as also seen experimentally. However, as long as the model outputs quickly converge to that of the system, and sufficient input data is used for determining the fault/disturbance distribution matrices, the obtained direction should be close to the desired optimum.

Table 1: comparison of optimization results.

\begin{tabular}{|c|c|c|}
\cline { 2 - 3 } \multicolumn{1}{c|}{} & $\begin{array}{c}\text { Criterion at the } \\
\text { exit of the } \\
\text { optimization }\end{array}$ & $\begin{array}{c}\text { Main optimization } \\
\text { parameters }\end{array}$ \\
\hline \hline LS method & 3.0834 & \\
\hline \multirow{2}{*}{$\begin{array}{c}\text { Nelder and } \\
\text { Mead } \\
\text { optimization } \\
\text { technique }\end{array}$} & 78.64 & $\mathrm{~N}=500$, TolX $=10^{-10}$ \\
\cline { 2 - 3 } & 9.6435 & $\mathrm{~N}=10^{4}$, TolX $=10^{-10}$ \\
\cline { 2 - 3 } & 9.6435 & $\mathrm{~N}=10^{5,}$ TolX $=10^{-10}$ \\
\cline { 2 - 3 } & 9.6435 & $\mathrm{~N}=10^{6,}$ TolX $=10^{-15}$ \\
\hline
\end{tabular}

Figure 3 shows the outputs of the system and of the model in the presence of fault 1 without decoupling (for details on decoupling techniques used, see Uppal \& Patton 2004). Note that the data correspond to different operating points. Figure 4 and 5 show the outputs of the model and of the system in the presence of fault 1 , the decoupling matrices being computed respectively by the LS formulation given in CASE III and a Nelder and Mead technique (with $\mathrm{N}=1000$ and TolX $=10^{-10}$ ).

It is quite evident that the results achieved in figure 4 are better than those obtained with the Nelder and Mead approach. Note that when $\mathrm{N}$ increases and TolX decreases, both plots seem to be equivalent. However, the norm of the error vector is much more smaller with the LS approach (see table 1). 

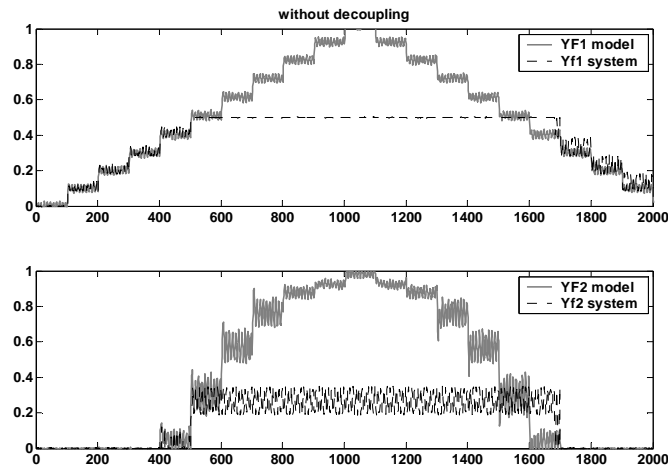

Fig. 3: Output of the system and of the model in presence of fault 1 without decoupling
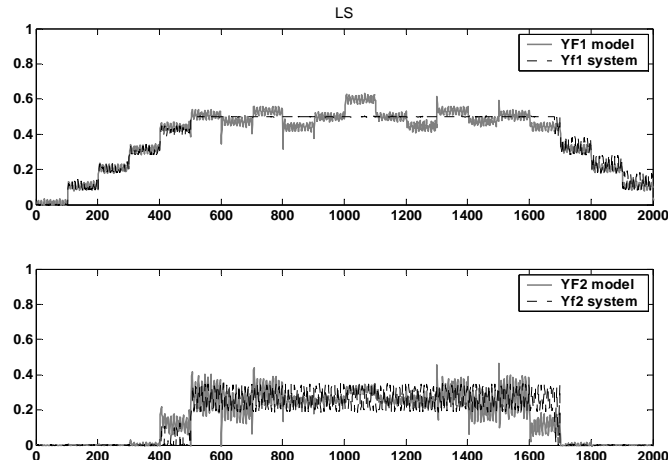

Fig. 4: System \& model outputs in presence of fault1 with decoupling using LS formulation
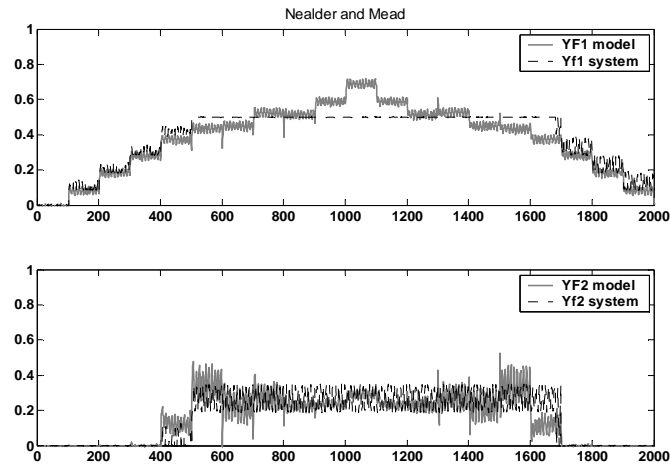

Fig. 5: System \& model outputs in presence of fault1 with decoupling using Nelder \& Mead optimization

Real data have been used with the NFDFDS scheme (Uppal \& Patton 2004; Uppal, Patton \& Witczak, 2004). However, this particular experiment with numerical improvement only involves simulated data.

\section{CONCLUSION}

This paper has given a new formulation of the computation of the disturbance and distribution matrices as a Least-Squares problem. It has been shown de novo that the original problem to be solved is linear in unknown parameters and consequently a Least-Squares approach is the most efficient compared with any other non-linear optimization technique. This facilitates the computation of the unique minimum L-2 norm solution when the LeastSquares matrix is rank deficient. The computational cost is greatly reduced compared with previous approaches. Hence, the LS approach allows an online update of the disturbance and distribution matrices. This is an important consideration in view of the apparent design and computational complexity of NF modelling and FDI methods, especially when integrated with the robust UIO problem.

\section{ACKNOWLEDGEMENTS}

The authors acknowledge funding support under the EC RTN contract (HPRTN-CT-2000-00110) DAMADICS and to the Dora Jones Trust of Hull University for funding support to Prof. Suzanne Lesecq whilst visiting University of Hull.

\section{REFERENCES}

Brown M \& Harris C J, (1995), Neuro-fuzzy adaptive modelling and control, Prentice Hall, ISBN 0131344536.

Chen J \& Patton R J (1996), Optimal filtering \& robust fault-diagnosis of stochastic systems with unknown disturbances, IEE Proc.-D: Contr.Theory \& Appl. 143 (1): 31-36.

Chen J \& Patton R J, (1999), Robust Model Based Fault Diagnosis For Dynamic Systems, Kluwer Academic Publishers ISBN 0-7923-8411-3.

DAMADICS Benchmark definition, ver.1.0. (2003), 'http://diag.mchtr.pw.edu.pl/damadics/', Institute of Automatic Control and Robotics - Warsaw University of Technology, accessed Dec 2004.

DAMADICS RTN Information Web site, (2003), 'http://diag.mchtr.pw.edu.pl/damadics', Institute of Automatic Control and Robotics - Warsaw University of Technology, last checked Dec 2004.

Dasgupta D \& Michalewicz Z, eds., (1997), Evolutionary Algorithms in Engineering Applications. SpringVerlag, New York, NY.

Hagan M T, Demuth H \& Beale M (1996), Neural Network Design, PWS Publishing Company, Boston, MA, ISBN 0-534-94332-2.

Jang J S R, Sun C T \& Mizutani E, (1997), Neuro-Fuzzy and Soft Computing, Prentice-Hall, Inc., New Jersey, ISBN 0-13-261066-3.

Lawson C \& Hanson R, (1995), Solving Least-Squares Problems, Prentice Hall, Englewood Cliffs, N. J., 1974 (2nd edition, Philadelphia, Society for Industrial \& Applied Mathematics, ISBN: 0898713560).

Patton R J, Frank P M \& Clark R N (eds), (1989), Fault Diagnosis in Dynamic Systems, Theory \& Applications, Prentice Hall, NY.

Uppal F J \& Patton R J, (2005), A hybrid fault diagnosis approach applied to electro-pneumatic valves in sugar process, Special Issue on Condition Monitoring, Int. J. of Adaptive Control and Signal Processing (to appear).

Uppal F J, Patton R J \& Witczak M, (2005), A neuro-fuzzy multiple-model observer approach to robust fault diagnosis: based on the DAMADICS benchmark, In Special Issue on: The DAMADICS Benchmark Problem Development and Application of Methods for Actuator Diagnosis in an Industrial Control Systems: The DAMADICS Benchmark Study, Int. J. Control Engineering Practice (accepted for publication).

Walsh G R (1975), Methods of Optimisation, John Wiley, Chichester, UK.

Witczak M, Korbicz J, Mrugalski M \& Patton R J, (2003), A GMDH neural network-based approach to robust fault diagnosis: Application to the DAMADICS benchmark problem, In Special Issue on: Development and Application of Methods for Actuator Diagnosis in an Industrial Control Systems: The DAMADICS Benchmark Study, Int. J. Control Engineering Practice (to be published in 2005). 